\title{
Design of class-E Power Amplifier (PA) with minimum harmonious termination for Cellular phones
}

\author{
Zahraa Mohammed younis saed ${ }^{1}$, Dr. Khalid Khaleel ${ }^{2}$ \\ \{Zahraaalslam530@gmail.com ${ }^{1}$, khalid.khaleel@uoninevah.edu.iq ${ }^{2}$ \} \\ Mosul University, Electrical Engineering Department ${ }^{1}$ \\ Nineveh University ${ }^{2}$
}

\begin{abstract}
Power efficiency is a critical element in the design of RF power amplifiers for contemporary wireless applications. Improved power efficiency results in longer battery life, less heat dissipation needs, a more compact design, and increased dependability. A high-efficiency Amplifier with class-E power is described in this article, which is based on a GaAs transistor and is optimized for operation in the $1.8 \mathrm{GHz}$ LTE band. It is possible to improve the efficiency and output power of a transmission line-based output network by decreasing the harmonic powers of the transmission lines. It is found that all harmonic level values down than $-90 \mathrm{dBc}$ were suppressed by the proposed class-E amplifier. With a 7-dB increase in output power, the peak power-added efficiency (PAE) is 82 percent at $30 \mathrm{dBm}$ output power, resulting in a power gain of 82 percent.
\end{abstract}

Keywords: Gallium Arsenide (GaAs), Amplifier of class-E power (PA), Harmonic suppression, power-added efficiency (PAE).

\section{Introduction}

Radiofrequency (RF) amplifiers are essential components in a wide range of communication systems, including satellite communications. All the application in point-to-point communication or wireless communication usually uses the advantages modern power amplifier, which is critical components. The working frequency of the amplifiers is determined by the application, and it may range from extremely low to microwave frequencies in the frequency range. In situations when a signal must be amplified to a known value at any application frequency, RF power amplifiers are used to achieve the desired results (PAs). The power outputs of the amplifiers may likewise range from milliwatts to megawatts in output. The most popular RF amplifiers are usually classified into the $\mathrm{A}, \mathrm{B}, \mathrm{AB}, \mathrm{C}, \mathrm{D}, \mathrm{E}, \mathrm{F}$, and $\mathrm{S}$ classes. In this section, we will show the amplification of a linear or nonlinear signal using several topologies. Nonlinear amplification is achieved by utilizing amplifiers with Class A, B, or $\mathrm{AB}$ amplifiers topologies; linear amplification is achieved by employing amplifiers with Class C, D, E, F, and S amplifiers topologies. The amplifier classes D, E, F, and S have been classified as switch-mode amplifiers because it performs the role of a switch during amplifier operation. The following is an explanation of the relationship between the RF signal forms amplifiers input and output: 
$v_{\mathbf{0}}(t)=\beta v i(t)$

Nonlinear operation of the amplifier results in the signals at the input and output being represented as a power series, as shown in Equation 2:

$v_{0}(t)=\alpha_{0}+\alpha_{1} v i(t)+\alpha_{2} v i^{2}(t)+\alpha_{3} v i^{3}(t)+\ldots$.

Equation 2 illustrates the amplifier response's modest nonlinearities. When there is just minor distortion [1].

\section{Class-E (PA)}

Sokal and Sokal [2] were the first to develop a class-E power amplifier (PA), which was introduced in 1975. According to the theory, the Class-E amplifier may achieve high efficiency because it prevents the simultaneous presence of nonzero switching voltage and current [3]-[6]. Amplifiers of class-E power and variants have been extensively employed in wireless communications. Amplifiers of class-E power PAs have theoretical efficiencies approaching $100 \%$ [7]. Its circuit comprises a switching transistor, a load network, and a load impedance. A shunt capacitor and a series resonant circuit comprise the load network [8]. Due to its great efficiency, a class-E power amplifier achieves very high dc to ac conversion efficiency at high frequencies, owing to the substantial decrease in switching losses. The switching power amplifier technology's fundamental concept is that the transistor enters a saturated state and switches on and off the voltage or current, depending on the kind of amplifier [4].

\section{Design and Simulation OF Class-E PA}

The design starts with the single-ended class-E PA selects GaAs MESFET transistor, GaAs MESFET makes the amplifiers at microwave frequencies. It is common for the bias voltage Vgs of a class-E transistor to be equal to the threshold voltage of the transistor to ensure that the transistor functions in the switch state. Construction of the basic circuit for a class-E power amplifier is then shown in Fig 1 There are three parts to this circuit: a direct current source $\mathrm{VDD}$, an RF choke Lf, and a load network that is composed of a series resonant $\mathrm{L}_{0} \mathrm{C}_{0}$ the resonance frequency of $\mathrm{L}_{0} \mathrm{C}_{0}$ will be the same as input frequency which means that the two frequency is tuned together, and a shunt capacitor Cs. 


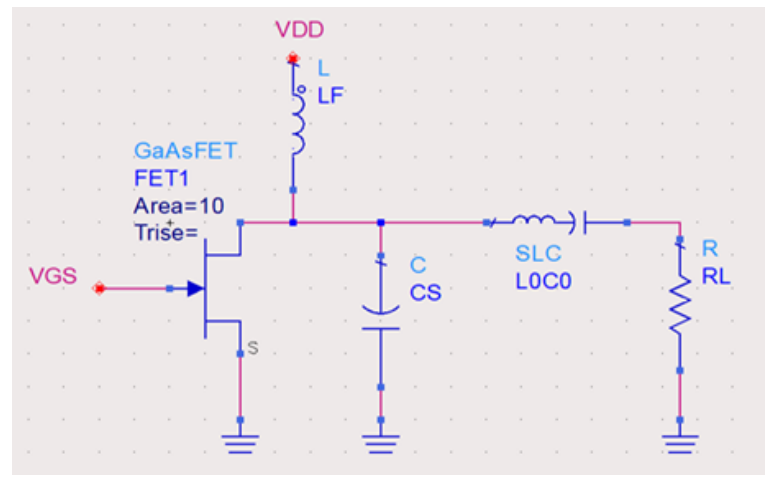

Fig.1. The basic circuit of a class-E PA.

Then designed output and input matching circuit, if we can control an unlimited number of harmonics in the amplifier output through an $\mathrm{LnCn}$ series resonator tuned to the nth harmonic, the amplifier's efficiency will approach $100 \%$. Significant control is feasible only for certain harmonics and only a few output harmonics will dominate. Using a series of tank LC circuit to generate resonance at a certain harmonic in the output spectrum and a load resistance $\mathrm{R}$ is an efficient method to do this. The values of the components in the circuit shown in Figure (1) (a parallel capacitance $\mathrm{Cs}$, a series resonant $\mathrm{L}_{0} \mathrm{C}_{0}$ circuit) are calculated at output power Pout $=32$ $\mathrm{dBm}$, operating frequency $\mathrm{f}_{0}=1.8 \mathrm{GHz}$, loaded quality factor $Q_{l}=10$ and $\mathrm{VDD}=4 \mathrm{~V}$ using the following equations [5] and theoretical value of this parameter shown in Table 1.

$R=\frac{8 V D D^{2}}{\pi^{2}+4 P_{o}} \approx 0.5768 \frac{V D D^{2}}{P_{o}}$

$X C_{S}=\frac{1}{\omega C_{S}}=\frac{\pi\left(\pi^{2}+4\right) R}{8} \approx 5.4466 R$

Where $\omega=2 \pi f_{o}$

$$
\begin{aligned}
& X L_{\mathrm{o}}=\omega L_{\mathrm{o}}=Q_{l} R \\
& X C_{\mathrm{o}}=\frac{1}{\omega C_{0}}=\left[Q_{l}-\frac{\pi\left(\pi^{2}-4\right)}{16}\right] R \approx\left(Q_{l}-1.1525\right) R
\end{aligned}
$$

Then, a matching circuit was built. After determining ZL and Zs via load-pull and source pull simulations and designing output and input matching circuits in ADS using the Smith chart tool, the Q-factor of inductor utilized to calculate matching circuit is equal to 10 in this case. It is the matching of outputs that is the core of class-E PA technology. Stability, gain, and other characteristics are significantly influenced by the input circuit. The output circuit, on the other hand, may modify the waveform and therefore increase the output power and efficiency. Then, for harmonic termination, an LnCn series resonator tuned to frequencies $2 f_{o}, 3 f_{o}, 4 f_{o}$, and $5 f_{o}$ was developed. All harmonic components should have an open circuit in the harmonic 
termination network. Because the output matching network does not open to all harmonics, in an ideal class-E architecture, the nonideal series resonant filter results in undesirable harmonics at the output, degrading the amplifier's performance. As a result, harmonic powers are suppressed, and losses are minimized. For class-B operation at $1.8 \mathrm{GHz}$, a class-E PA was constructed using GaAs with a $P_{o}=30 \mathrm{dBm}$, a gain of $7 \mathrm{~dB}$, and power-added efficiency (PAE) of 82 percent at $4 \mathrm{~V}$. The class-E PA is designed using the following formulae (3)-(6) to determine the value of each load network component. Table (1) contains the values for the elements.

Table1.shows the element values of the load network.

\begin{tabular}{lll}
\hline Parameter & Value & Unit \\
\hline $\begin{array}{l}\text { operating } \\
\text { frequency } \mathrm{f}_{\mathrm{o}}\end{array}$ & 1.8 & $\mathrm{GHz}$ \\
$\mathrm{R}$ & 5.8 & $\Omega$ \\
$\mathrm{L}_{\mathrm{o}}$ & 5.5 & $\mathrm{nH}$ \\
$\mathrm{C}_{\mathrm{o}}$ & 1.723 & $\mathrm{pF}$ \\
$\mathrm{Cs}$ & 2.798 & $\mathrm{pF}$ \\
\hline
\end{tabular}

It should be noted that the values of the designed circuit elements have undergone a tuning software process in ADS to obtain the best results, and then design input and output matching circuit and harmonious termination circuit, the input matching elements of L4 and C5 were $0.5 \mathrm{nH}$ and $1.5 \mathrm{pF}$, respectively. And C6, L5 was $3.9 \mathrm{pF}$ and $1.5159 \mathrm{nH}$ respectively represent output matching circuit, and four-stage $\mathrm{LnCn}$ series resonator represents harmonious termination circuit, after implementation the power amplifier circuit we convert all the amplifier components to micro- strips line, because at high frequencies, the effect of stray capacitors and inductors is noticeably visible, as well as the poor performance of capacitors and inductors that are used in amplification circuits. Micro strips are one of the types of transmission lines that are used to transfer power from one point to another with high efficiency. The design is based on an ideal schematic of a microstrip circuit, and we must convert it into a real microstrip circuit to use it effectively. Because the dielectric constant is 4.3 , the dielectric substratum is used in this study as the dielectric substratum. The thickness of the substrate is $h=1.6 \mathrm{~mm}$. ADS is an acronym that stands for Automated Data Collection. The electric length E and characteristic impedance $\mathrm{Z}_{\mathrm{o}}$ converted into actual length $\mathrm{L}$ and width $\mathrm{W}$ of the micro-strip line by Linecalc module was called were $Z_{o}$ equal $50 \Omega$.

\section{Simulation Results}


The proposed power amplifier circuit Fig 2 has been modelled with the help of the ADS software package's harmonic balancing simulator with $P_{o}=30 \mathrm{dBm}$ with $7 \mathrm{~dB}$ and 82 percent power-added efficiency (PAE) gains at $1.8 \mathrm{GHz}$.

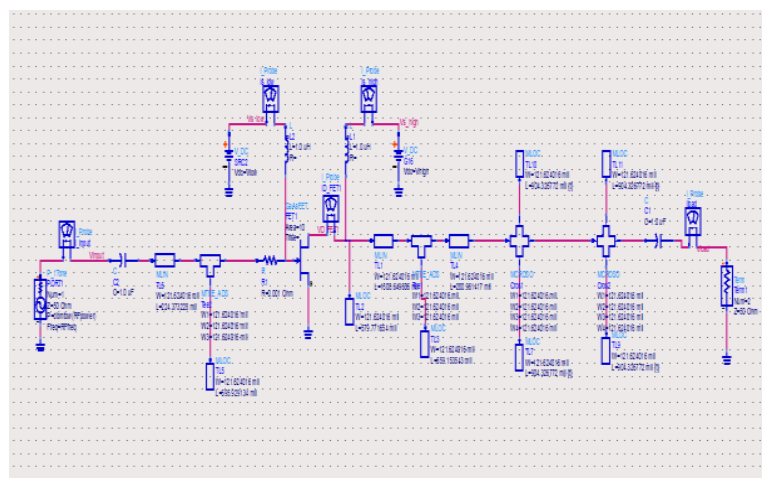

Fig.2. a complete micro-strip line circuit for a class-E power amplifier.

Fig 3 depicts the drain voltage and current waveforms of a GaAs transistor operating at $1.8 \mathrm{GHz}$. It is approximate approximation of half-wave sinusoidal pulses that are used in the simulation of drain voltage and current waveforms. As illustrated in figure [3], the drain current waveform should be out of phase with the drain voltage waveform with the least amount of overlap. This non-overlapping behaviour minimizes power dissipation in the GaAs transistor's drain, thus increasing the power amplifier's efficiency.

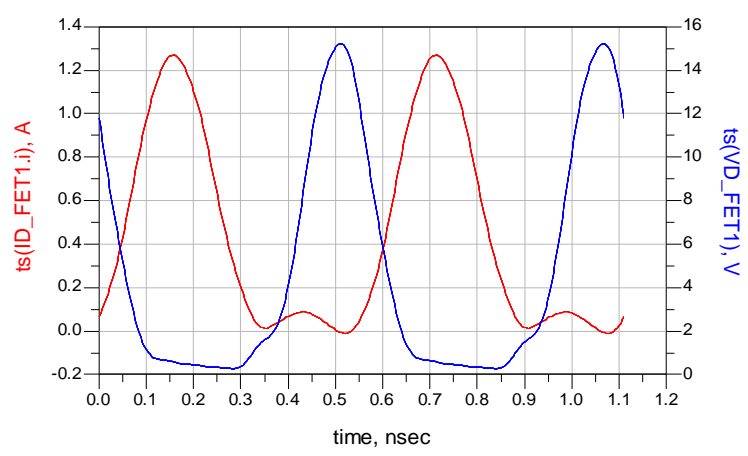

Fig.3. Waveforms of drain voltage and drain current. 
The output voltage waveform of the power amplifier circuit is shown in Fig 4. The sinusoidal shape of this waveform is due to the output matching network's low pass filtering effect, which reduces the amplitudes of the harmonic components.

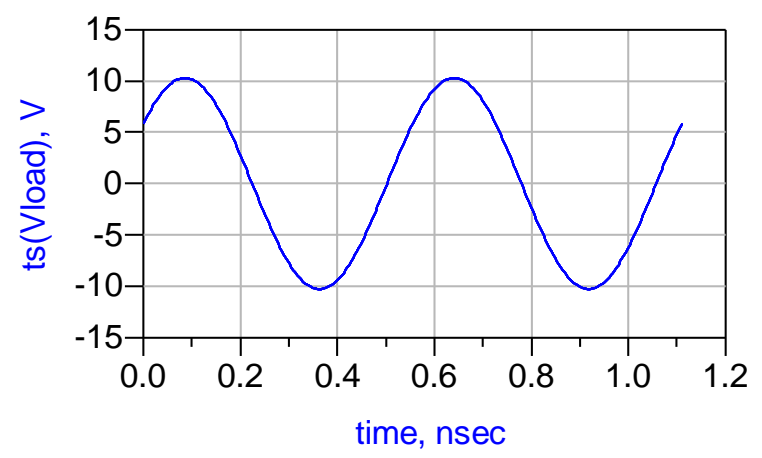

Fig.4. output voltage waveform.

The output spectrum waveform of the power amplifier circuit is sketched in Fig [5] for fundamental harmonics, it can be shown that the second and third harmonics are well suppressed to -70dBm compared to the fundamental harmonic which mean that the out of band noise is reduced to a minimum value.

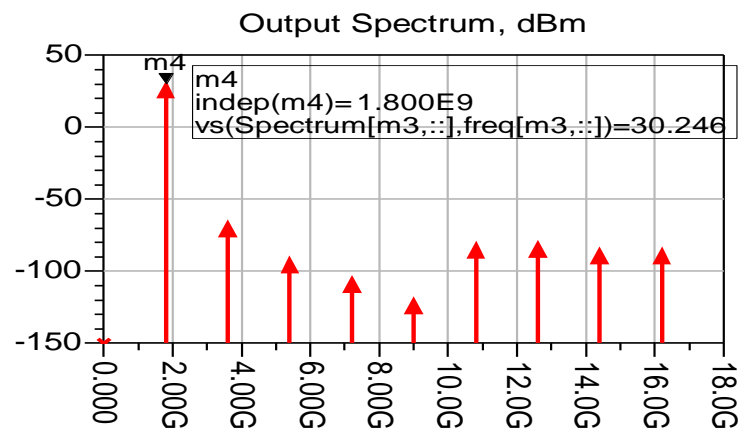

Fig.5.The output spectrum of the power amplifier circuit.

Result optimised can be is comfortable with another research and this same to be very good in the value of power added efficiency-optimised and suppressing second and third harmonic powers and with minimum overlap between current and voltage drain. 


\section{Conclusion}

A high-efficiency class-E PA circuit based on a GaAs MOSFET was developed and constructed in this work to enhance the overall performance and increase the efficiency by suppressing second and third harmonic powers as much as feasible. The harmonic power levels of the power amplifier circuit utilized for a single tone were kept below $-90 \mathrm{dBc}$ throughout the output power range of 24 to $30 \mathrm{dBm}$ using the single tone circuit. It was possible to achieve a peak PAE of 82 percent with a power gain of $7 \mathrm{~dB}$ for an output power of $30 \mathrm{dBm}$ while operating at $1.8 \mathrm{GHz}$ and therefore achieve a power gain of $7 \mathrm{~dB}$ for the output power.

\section{References}

[1] EROGLU, Abdullah. Introduction to RF power amplifier design and simulation . CRC press, 2018. Chapter one, Radio Frequency Amplifier Basics; p. [1].

[2] N. O. Sokal and A. D. Sokal. "Class E-A new class of high-efficiency tuned single-ended switching power amplifiers”, IEEE Journal of SolidState Circuits, SC-10, pp. 168-176, June 1975.

[3] LENG, Yongqing, et al. An extended topology of parallel-circuit Amplifier of class-E power using transmission-line compensation. IEEE transactions on microwave theory and techniques, 2013, 61.4: 1628-1638.

[4] YUSMARNITA, Y., et al. Design and analysis of 1MHz Amplifier of class-E power. WSEAS Journal, 2015, 14.

[5] Kazimierczuk, M. K. (2008). RF power amplifiers . (Vol. 1). New York:: Wiley.Chapter five, Class E Zero-Voltage Switching RF Power Amplifiers ;p.[255].

[6] LIU, Chang; CHENG, Qian-Fu. Analysis and design of high-efficiency parallel-circuit class-E/F power amplifier. IEEE Transactions on Microwave Theory and Techniques, 2019, 67.6: 2382-2392.

[7] WANG, Jingyuan; YU, Hongxi; YANG, Fei. Design of GaN HEMT class-E power amplifier for satellite communication. In: 2018 International Conference on Microwave and Millimeter Wave Technology (ICMMT). IEEE, 2018. p. 1-3.

[8] RAAB, Frederick. Idealized operation of the class E tuned power amplifier. IEEE transactions on Circuits and Systems, 1977, 24.12: 725-735. 